\title{
A semi-automated FISH-based micronucleus-centromere assay for biomonitoring of hospital workers exposed to low doses of ionizing radiation
}

\author{
ANNE VRAL ${ }^{1}$, VEERLE DECORTE $^{2}$, JULIE DEPUYDT $^{1}$, ANDRÉ WAMBERSIE $^{3}$ and HUBERT THIERENS $^{1}$ \\ ${ }^{1}$ Department of Basic Medical Sciences, Ghent University, B-9000 Ghent; \\ ${ }^{2}$ Scientific Committee Centre de Services Interentreprises, External Service For Prevention and Protection at Work; \\ ${ }^{3}$ Institute of Experimental and Clinical Research, Molecular Imaging, Radiotherapy and Oncology Unit, \\ Catholic University of Louvain, St-Luc Clinical University, B-1200 Brussels, Belgium
}

Received October 22, 2015; Accepted March 10, 2016

DOI: $10.3892 / \mathrm{mmr} .2016 .5265$

\begin{abstract}
The aim of the present study was to perform cytogenetic analysis by means of a semi-automated micronucleus-centromere assay in lymphocytes from medical radiation workers. Two groups of workers receiving the highest occupational doses were selected: 10 nuclear medicine technicians and 10 interventional radiologists/cardiologists. Centromere-negative micronucleus (MNCM-) data, obtained from these two groups of medical radiation workers were compared with those obtained in matched controls. The blood samples of the matched controls were additionally used to construct a 'low-dose' (0-100 mGy) MNCM- dose-response curve to evaluate the sensitivity and suitability of the micronucleus-centromere assay as an 'effect' biomarker in medical surveillance programs. The physical dosimetry data of the 3 years preceding the blood sampling, based on single or double dosimetry practices, were collected for the interpretation of the micronucleus data. The in vitro radiation results showed that for small sized groups, semi-automated scoring of MNCM- enables the detection of a dose of $50 \mathrm{mGy}$. The comparison of MNCM- yields in medical radiation workers and control individuals showed enhanced MNCM- scores in the medical radiation workers group $(\mathrm{P}=0.15)$. The highest MNCM- scores were obtained in the interventional radiologists/cardiologists group, and these scores were significantly higher compared with those obtained from the matched control group $(\mathrm{P}=0.05)$. The higher MNCM- scores observed in interventional radiologists/cardiologists compared with
\end{abstract}

Correspondence to: Professor Anne Vral, Department of Basic Medical Sciences, Ghent University, Heymans Campus, University Hospital, Building 6B3, 185 De Pintelaan, B-9000 Ghent, Belgium E-mail: anne.vral@ugent.be

Key words: micronucleus assay, centromeres, DNA damage, ionizing radiation, low dose exposure, hospital workers, biomonitoring, dosimetry nuclear medicine technicians were not in agreement with the personal dosimetry records in both groups, which may point to the limitation of 'double dosimetry' procedures used in interventional radiology/cardiology. In conclusion, the data obtained in the present study supports the importance of cytogenetic analysis, in addition to physical dosimetry, as a routine biomonitoring method in medical radiation workers receiving the highest occupational radiation burdens.

\section{Introduction}

Of all the workers exposed worldwide to man-made sources of ionizing radiation, medical radiation workers represent the largest group. Although the exposure levels in these subjects are generally low and in any case below the regulatory limit of $20 \mathrm{mSv}$ per year, diagnostic procedures used in nuclear medicine and interventional cardiology/radiology can still represent a source of relatively high occupational exposure $(1,2)$.

In nuclear medicine the highest exposures occur during the handling and administration to patients of fluorine-18 fluorodeoxyglucose (18F-FDG) for positron emission tomographic (PET) imaging. In the medical application of X-rays, the highest exposures are associated with interventional procedures in interventional radiology/cardiology, necessitating fluoroscopy and cinefluorographic examinations. The occupational dose of staff working in interventional radiology/cardiology remains high despite the wearing of lead aprons. Workers receive doses from scattered radiation and the transmission of a small percentage of primary x-rays through the apron, and additionally, part of the body is unshielded. In interventional cardiology, the continuous developments in catheter device technology and procedures enable more challenging clinical indications to be handled. As a result, the number of interventional procedures, in addition to their complexity, increases together with the potential for interventional operators to receive higher doses of ionizing radiation (3). This raises some concern as epidemiological studies have indicated that medical radiation workers may show increased mortality due to leukemia and other neoplasias (4). 
Ionizing radiation is a potent mutagen and inducer of chromosomal aberrations, which are correlated with genetic alterations that may trigger the development of cancer. Chromosomal damage in peripheral blood lymphocytes induced by ionizing radiation can be assessed by cytogenetic techniques, such as the dicentric assay and the micronucleus assay. Cytogenetic studies performed on hospital workers occupationally exposed to low doses of ionizing radiation have indicated that even in cases where the levels of radiation received through occupational exposure were under regulatory limits, as registered by personal dosimetry, increased cytogenetic damage was observed, compared with the non-exposed subjects. By using chromosomal damage as 'effect' biomarker, cytogenetic biomonitoring provides important additional information which complements physical dosimetry and enables better evaluation of the health effects of radiation $(1,2,4-7)$.

Although cytogenetic analysis of dicentric formation is the gold standard for biological dosimetry, application of this technique for the biomonitoring of relatively large groups of radiation workers is not ideal, as dicentric analysis is complex, time consuming and requires highly skilled personnel. By contrast, the cytokinesis-block micronucleus (MN) assay is less laborious and presents a viable alternative for large-scale screening. The only disadvantage of this assay compared with the dicentric assay is the high frequency of background spontaneous micronuclei, making this technique less sensitive for the detection of low dose exposures (detection limit of 0.2-0.3 Gy) (8). However, combination of the MN assay with fluorescent in situ hybridisation (FISH) using a pan-centromeric probe enables discrimination between radiation-induced centromere-negative MN (MNCM-), resulting mainly from acentric fragments, and spontaneous centromere-positive $\mathrm{MN}$ (MNCM+), which predominantly contain lagging chromosomes. Scoring of MNCM- has substantially increased the sensitivity of the technique for the biomonitoring of radiation workers (4). Although the FISH-based MN-centromere assay is more expensive and time consuming, new developments in FISH technology and the availability of computerized cytogenetic image analysis systems have overcome this problem. The semi-automated MN-centromere assay recently optimized by our research group combines high-speed $\mathrm{MN}$ analysis with a more accurate assessment of radiation damage in the low dose range (0.05-0.1 Gy), comparable to the dicentric assay. These characteristics of the semi-automated MN-centromere assay are of special interest for large-scale radiation exposure applications (9).

The aim of the present study was to perform a cytogenetic analysis by means of the semi-automated MN-centromere assay in the peripheral blood lymphocytes (PBL) of medical radiation workers occupationally exposed to low doses of ionizing radiation (below the regulatory limits). Two groups of medical workers that receive the highest doses at their workplace were selected: Nuclear medicine technologists (NMTs) involved in PET applications and interventional cardiologists/radiologists. The MN data obtained from these workers were compared with a group of non-exposed, matched control individuals. The blood samples of the control individuals were additionally used to construct a 'low dose' (0-100 mGy) MN dose-response curve to evaluate the sensitivity of the MN assay in the low dose range and its suitability as bio-dosimeter in medical surveillance programs. For both groups of radiation workers, physical dosimetry data were collected for the interpretation of the MN data: Personal equivalent dose $\mathrm{Hp}(10)$ values for the nuclear medicine technicians and effective dose $\mathrm{E}$ values for the interventional X-ray workers. The results from the present study support the importance of cytogenetic analysis, in addition to physical dosimetry, as a routine biomonitoring method in medical radiation workers with the highest occupational radiation burdens.

\section{Materials and methods}

Study population. For the present study two groups of medical radiation workers were selected: 10 NMTs and 10 interventional radiation workers (IRW). The NMT group was recruited from the following hospitals: University Hospital St-Luc, Iris Hospitals South, St-Anne St-Rémi Clinic (all Brussels, Belgium) and AZ St-Jan (Bruges, Belgium). The IRW group consisted of cardiologists and radiologists working in the following hospitals: Mont-Godinne Clinic (Yvoir, Belgium), Brugmann University Hospital (Brussels, Belgium), Hospital Oost-Limburg, Ghent University Hospital (Ghent, Belgium) and St-Luc (Brussels, Belgium). Two control groups, one for each category of exposed medical radiation workers $\left(\mathrm{CON}_{\mathrm{NMT}}\right.$ and $\mathrm{CON}_{\text {IRW }}$ ), were comprised of age- and gender-matched individuals and included 19 subjects working in the same hospitals, however, with no history of exposure to ionizing radiation.

The study was approved by the ethics committee of St-Luc Clinical University (Brussels, Belgium). All participants were volunteers and each individual was provided with sufficient information to give an informed consent. None of the participants had undergone X-ray or isotope examinations during the previous 5 years, or had been exposed to other carcinogenic or mutagenic agents at work. None of the participants had experienced major medical problems or conditions during the previous 5 years. There was one smoker in the exposed group and one in the control group. The mean age of the NMT group, consisting of 4 males and 6 females, was 46.4 years (range: 26-64) compared with 45.7 years (range: $31-62$ ) for the corresponding control group. The mean age of the IRW group (10 males) was 47.8 years (range: 41-60) compared with 47.5 years (range: $42-56$ ) for the controls. The age of the participants in the study ranged from 26-64 years. Heparinized blood samples from the radiation workers and matched controls were taken by venipuncture at the same time. Blood samples of the worker/control pairs were collected and coded in the Occupational Medicine Service, Centre de Services Interentreprises (CESI; University Hospital St-Luc, Iris Hospitals South, St-Anne St-Rémi Clinic and Mont-Godinne Clinic) and sent to the biodosimetry laboratory at Ghent University (Ghent, Brussels) for cytogenetic analysis.

Dosimetry records. The occupational radiation doses received in the years prior to the blood sampling performed in the present study had been routinely monitored by film badge personal dosimetry. In the case of nuclear medicine workers the dose estimates, $\mathrm{Hp}(10)$, are based on the monthly readings from personal dosimeters fixed at chest height to the hospital apron of the workers. Determination of the radiation doses of the IRWs 
was performed by double dosimetry to compensate for the fact that part of the body is shielded by the lead apron and part of the body remains unshielded. One dosimeter was worn underneath the lead apron at chest or waist height, resulting in a dose under the apron, Hp,u(10), and the other is worn above the apron at chest height, resulting in a dose above the apron, Hp,o(10). The readings were combined to obtain the effective dose $\mathrm{E}$ using the algorithm $\mathrm{E}=0.79 \mathrm{Hp}, \mathrm{u}(10)+0.100 \mathrm{Hp}, \mathrm{o}(10)(10)$. This algorithm takes into account organs and weighting factors from the International Commission on Radiological Protection (11).

Personal dosimetry records for the 3 years preceding the blood sampling were collected for each radiation worker belonging to the study cohort. Dosimetry records older than 3 years were not collected, as unstable chromosome aberrations such as dicentrics and MN have an in vivo half-life of approximately 1 year. In addition, Thierens et al (12) reported that chromosomal damage induced greater than 3 years ago had almost completely disappeared.

\section{Semi-automated MN centromere assay}

Cell culture and MN-centromere assay. The day of blood sampling, two $5 \mathrm{ml}$ whole blood cultures were set up per sample containing $0.3 \mathrm{ml}$ blood in Roswell Park Memorial Institute 1640 medium with L-glutamine and $25 \mathrm{mM}$ 4-(2-hydroxyethyl)-1-piperazineethanesulfonic acid buffer (HEPES) (Gibco; Thermo Fisher Scientific, Inc., Waltham, MA, USA) supplemented with $10 \%$ fetal calf serum (Gibco; Thermo Fisher Scientific, Inc.) and $100 \mu \mathrm{l}$ phytohaemagglutinin (PHA; stock solution $1 \mathrm{mg} / \mathrm{ml}$ ) (Sigma-Aldrich, St Louis, MO, USA) used as a mitogen. At $23 \mathrm{~h}$ later, cytochalasin B (6 $\mu \mathrm{g} / \mathrm{ml}$, Sigma-Aldrich) was added to block cytokinesis. Cells were harvested $70 \mathrm{~h}$ following stimulation with PHA using a cold $\left(4^{\circ} \mathrm{C}\right)$ hypotonic shock with $0.075 \mathrm{M} \mathrm{KCl}$ (Merck Millipore, Darmstadt, Germany). This was followed by fixation in a solution containing methanol, acetic acid and ringer $(0.9 \% \mathrm{NaCl})(4: 1: 5)$ and a further fixation in methanol, acetic acid (4:1; Merck Millipore) for three times (13). Cell suspensions were added dropwise onto clean slides. All slides were prepared in duplicate per culture and coded. For the analysis of centromere-negative $\mathrm{MN}$, slides were dehydrated in alcohol series (70-90-100\% ethanol) (Merck Millipore) and left to dry for 15 min prior to performing FISH with a home-tailored probe based on PCR technology. The details of the pan-centromeric probe production, dilution and labelling [Nick Translation method, Spectrum Orange (SpOr)] have been previously described in detail (9). For in situ hybridisation, $20 \mu 1$ of the home-tailored probe was placed onto a coverslip and applied to the slide. Probe and target DNAs were then denatured at $76^{\circ} \mathrm{C}$ for $5 \mathrm{~min}$ and hybridised for $20 \mathrm{~h}$ at $38^{\circ} \mathrm{C}$ using a ThermoBrite Temperature controlled slide processing system (Abbott Molecular, Des Plaines, IL, USA). Following hybridisation, the slides were placed in $2 \mathrm{X}$ saline sodium citrate (SSC) for $5 \mathrm{~min}$ to allow detachment of the coverslip. Post-hybridisation washing was performed in $0.4 \mathrm{X} \mathrm{SSC} / 0.1 \%$ Tween at $72^{\circ} \mathrm{C}(1 \mathrm{~min})$ and in $2 \mathrm{X} \mathrm{SSC}$ at room temperature $(5 \mathrm{~min})$. Finally, the slides were mounted with Vectashield containing 4',6-diamidino-2-phenylindole (DAPI) (Vector Laboratories, Inc., Burlingame, CA, USA).

Blood samples from 16 of the 19 control individuals were used to set up an in vitro 'low-dose' dose-response curve. For this, each blood sample was divided in four parts. One part was used as a sham-irradiated control while the others were exposed to 20,50 and $100 \mathrm{mGy}$ of ${ }^{60} \mathrm{Co} \gamma$-rays at a dose rate of $0.1 \mathrm{~Gy} / \mathrm{min}$. For each dose point, two cultures were set up and processed as described above for $\mathrm{MN}$-centromere analysis.

Semi-automated MN-centromere scoring. Microscopic analysis was conducted using a Metafer 4 platform (MetaSystems $\mathrm{GmbH}$, Altlussheim, Germany) connected to a motorized Zeiss AxioImager M1 microscope (Zeiss, Oberkochen, Germany). Detailed information regarding the MSearch slide scanning procedure, stage movement, focusing and image acquisition are detailed in Willems et al (13) and Schunck et al (14).

First, automated MN scoring in binucleate (BN) cells on DAPI-FISH stained slides was performed using a 10x objective, a DAPI filter and the Msearch and MNScore modules of MetaSystems (13). Secondly, all BN cells that were detected by MSearch were visually checked in the image gallery and BN cells with confirmed MN were marked for AutoCapt analysis. With the AutoCapt image acquisition software, the marked cells were relocated from the Metafer position list using a 40x objective and image acquisition was performed with two colour channels (DAPI, SpOr). The two colour images were shown on the display and in the image gallery, and the MN were manually checked for the presence of centromeres. Fig. 1 presents an example of a $\mathrm{BN}$ cell with a centromere-positive $\mathrm{MN}(\mathrm{MNCM}+)$ containing a whole chromosome and a $\mathrm{BN}$ cell with a centromere-negative MN (MNCM-) consisting of an acentric fragment.

Semi-automated analysis of MN in FISH-DAPI stained slides, as described above, was performed to score the total number of $\mathrm{MN}$ (MN total), the number of MNCM- and $\mathrm{MNCM}+$ in the non-irradiated and irradiated samples from the workers and healthy controls. Between 2,000 and 6,000 $\mathrm{BN}$ cells were scored per condition.

Statistical analysis. All MN values are presented as the mean per $1,000 \mathrm{BN}$ cells \pm standard error of the mean. The personal dosimetry data is presented as the mean $(\mathrm{mSv}) \pm$ standard deviation. Statistical analysis of the data was performed using SPSS software, version 23.0 (IBM SPSS, Armonk, MY, USA). Differences in the MN yields between in vitro doses were analysed by a Wilcoxon signed-rank test (intra-group analysis). For difference in MN yield between two or more groups the Wilcoxon rank-sum test and the Kruskal-Wallis test were used, respectively. Agreement between dose-response fits and experimental data was quantified by the regression coefficient $\mathrm{R}^{2} . \mathrm{P} \leq 0.05$ was considered to indicate a statistically significant difference.

\section{Results}

In vitro $M N$ dose response curves. Blood samples from the 16 healthy control individuals (10 males, 6 females) were used to set up a dose-response curve in the low dose region. The mean dose-response data for MN total, MNCM- and MNCM+ are presented in Table I.

In Fig. 2, the linear-quadratic dose-response curves $\left(\mathrm{Y}=\mathrm{c}+\mathrm{aD} \mathrm{bD}^{2}\right.$ in which $\mathrm{Y}$ is the $\mathrm{MN}$ yield, $\mathrm{c}$ is the spontaneous $\mathrm{MN}$ count and $\mathrm{D}$ is the dose in $\mathrm{mGy}$ ) 
Table I. Total number of BN cells scored per dose for the 16 control individuals together with the mean MN scores and SEM for $\mathrm{MN}$ total (per 1,000 BN cells), MNCM- and MNCM+.

\begin{tabular}{|c|c|c|c|c|c|c|c|c|c|}
\hline Dose (mGy) & Total BN & MN total & SEM & $\begin{array}{l}\text { MNCM- } \\
\text { (per } 1,000 \\
\text { BN cells) }\end{array}$ & SEM & $\begin{array}{c}\text { MNCM- } \\
(\%)\end{array}$ & $\begin{array}{c}\text { MNCM+ } \\
\text { (per } 1,000 \\
\text { BN cells) }\end{array}$ & SEM & $\begin{array}{c}\text { MNCM+ } \\
(\%)\end{array}$ \\
\hline 0 & 52252 & 12.84 & 1.53 & 2.65 & 0.29 & 21 & 10.19 & 1.45 & 79 \\
\hline 20 & 50301 & 13.74 & 1.22 & 3.30 & 0.31 & 24 & 10.44 & 1.19 & 76 \\
\hline 50 & 49964 & 14.36 & 1.29 & 4.27 & 0.50 & 30 & 10.09 & 1.17 & 70 \\
\hline 100 & 46945 & 18.18 & 1.17 & 6.68 & 0.50 & 37 & 11.50 & 1.05 & 63 \\
\hline
\end{tabular}

BN, binucleate; MN, micronuclei; SEM, standard error of the mean; MNCM-, centromere-negative MN yields; MNCM, centromere-positive MN yields.

Table II. Mean MN yields for the groups of exposed workers compared with the matched control groups.

\begin{tabular}{|c|c|c|c|c|c|c|c|c|c|}
\hline \multirow[b]{2}{*}{ MN yields } & \multicolumn{3}{|c|}{ All, n=19 } & \multicolumn{3}{|c|}{ IRW, $\mathrm{n}=10$} & \multicolumn{3}{|c|}{ NMT, n=9 } \\
\hline & Exposed & $\mathrm{CON}$ & $\mathrm{P}$-value & Exposed & $\mathrm{CON}$ & P-value & Exposed & $\mathrm{CON}$ & P-value \\
\hline MN total ${ }^{\mathrm{a}}$ & 13.71 & 12.02 & 0.30 & 12.02 & 8.39 & 0.06 & 15.41 & 15.28 & 0.85 \\
\hline MNCM- $^{a}$ & $3.34(24)$ & $2.51(21)$ & 0.15 & 3.99 (33) & $2.44(29)$ & 0.05 & $2.70(18)$ & $2.58(17)$ & 0.68 \\
\hline $\mathrm{MNCM}+^{\mathrm{a}}$ & $10.37(76)$ & $9.51(79)$ & 0.63 & $8.03(67)$ & $5.95(71)$ & 0.20 & $12.71(82)$ & $12.70(83)$ & 1.00 \\
\hline
\end{tabular}

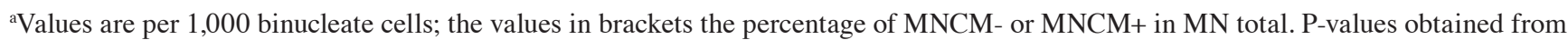
the Wilcoxon rank-sum test. MN, micronuclei; IRW, interventional radiation workers; NMT, nuclear medicine technologists; CON, controls; MNCM-, centromere-negative MN yields; MNCM+, centromere-positive MN yields.

Table III. Personal dosimetry data for the IRW and NMT groups presented as cumulative doses over periods of 12 and 36 months preceeding the blood sampling.

\begin{tabular}{lrrrr}
\hline Group & Dose estimates & 12 months & 36 months & 36 months (corrected) \\
\hline IRW $(\mathrm{n}=10)$ & $\mathrm{E}(\mathrm{mSv}) \pm \mathrm{SD}$ & $1.92 \pm 1.37$ & $6.03 \pm 4.71$ & $2.61 \pm 1.81$ \\
$\mathrm{NMT}(\mathrm{n}=9)$ & $\mathrm{Hp}(10)(\mathrm{mSv}) \pm \mathrm{SD}$ & $4.95 \pm 2.00$ & $17.02 \pm 7.01$ & $7.07 \pm 2.39$ \\
\hline
\end{tabular}

The 36 months (corrected) values are the cumulative doses over 36 months, corrected for the disappearance of micronucleated cells with a half-life of 1 year. IRW, interventional radiation workers; NMT, nuclear medicine technologists; SD, standard deviation.

that were fitted through the mean $\mathrm{MN}$ total (MN total $\left.=13.0+1.15 \times 10^{-2} \mathrm{D}+3.98 \times 10^{-4} \mathrm{D}^{2} ; \mathrm{R}^{2}=0.988\right)$ and MNCM- values (MNCM- $=2.68+2.53 \times 10^{-2} \mathrm{D}+1.46 \times 10^{-4} \mathrm{D}^{2}$; $\left.\mathrm{R}^{2}=0.999\right)$ are presented. Table I and Fig. 2 present an increase in the total number of $\mathrm{MN}$ in the $0-100 \mathrm{mGy}$ dose region, which may be attributed to an increase in MNCM-.

The yield of MNCM+ remains constant up to $50 \mathrm{mGy}$, with a slight increase at a dose of $100 \mathrm{mGy}$. Additionally, the percentages of MNCM- and MNCM+ are presented in Table I and show that spontaneous MN are predominantly centromere-positive (79\%). With increasing doses, the percentage of $\mathrm{MNCM}+$ reduced while the percentage of MNCM- increased, from $21 \%$ at 0 Gy to $37 \%$ at $100 \mathrm{mGy}$. A Wilcoxon signed-rank test was used to determine from which dose threshold significantly higher $\mathrm{MN}$ yields are obtained in our control group. For MNCM- a significant increase $(\mathrm{P}=0.032)$ was observed between 0 and $50 \mathrm{mGy}$, while for $\mathrm{MN}$ total a significant increase $(\mathrm{P}=0.003)$ was observed between 0 and $100 \mathrm{mGy}$. When MNCM- were analysed, a significant increase was observed between $50 \mathrm{mGy}$ and $100 \mathrm{mGy}(\mathrm{P}=0.004)$.

MN data in medical radiation workers compared with matched control individuals. A direct comparison of the mean values of MN total, MNCM- and MNCM+ between the two groups of radiation workers $(n=19)$ and their matched control groups $(n=19)$ is presented in Table II. In addition, the MNCM- data are presented graphically in Fig. 3. The IRWs exhibited increased but not significant $(\mathrm{P}=0.06)$ total $\mathrm{MN}$ yields, compared with their control group $\left(\mathrm{CON}_{\mathrm{IRW}}\right)$. With regards to MNCM-, the difference in $\mathrm{MN}$ yield between both groups was significant $(\mathrm{P}=0.05$; Table II, Fig. 3). As the individuals in both groups are age- and gender-matched, an influence of these variables in 
A

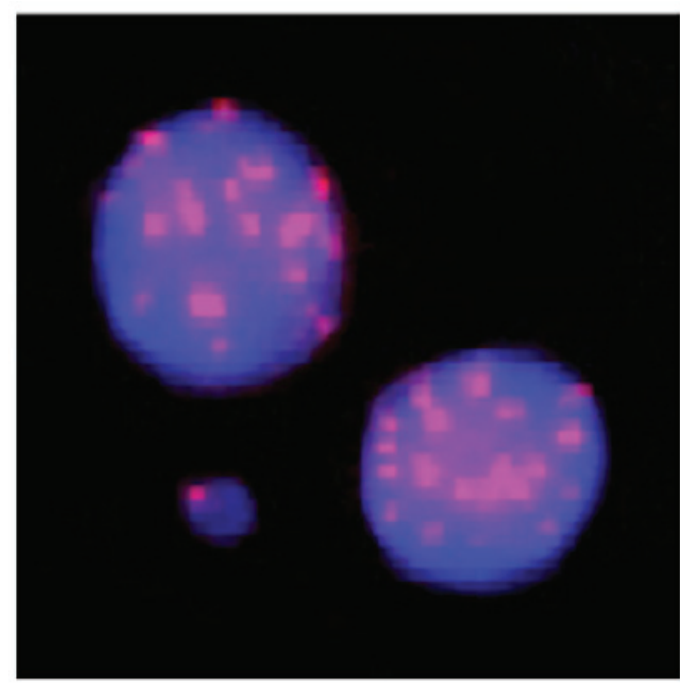

B

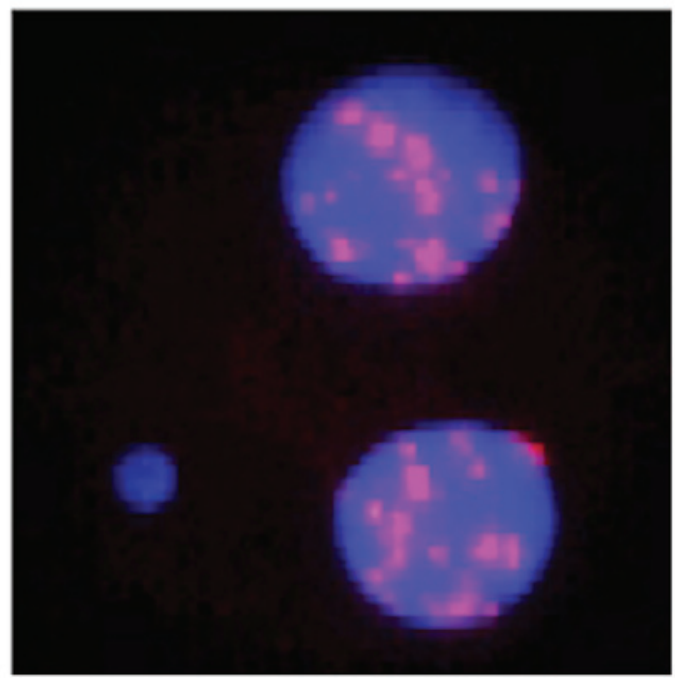

Figure 1. Images of BN cells. (A) The BN cell contains a centromere-positive MN. (B) The BN cell contains a centromere-negative MN (no signal). Nuclei are counterstained with 4',6-diamidino-2-phenylindole (magnification, x40). BN, binucleate; MN, micronuclei.

assessed MN scores may be ruled out. In the NMT group, no differences in $\mathrm{MN}$ yields ( $\mathrm{MN}$ total, MNCM-, MNCM+) were observed in comparison with their matched control group $\left(\mathrm{CON}_{\mathrm{NMT}}\right.$ ) (Table II, Fig. 3).

Comparison of the four populations (the IRW and NMT groups with their corresponding control groups) using a Kruskal-Wallis test indicated a significant difference in $\mathrm{MN}$ $(\mathrm{P}=0.02)$ and $\mathrm{MNCM}+(\mathrm{P}=0.01)$, however, not in MNCM$(\mathrm{P}=0.17)$. Histogram analysis indicates that the significant differences are predominantly associated with low $\mathrm{MN}$ and $\mathrm{MNCM}+$ values in the IRW control group.

Comparison of all radiation workers compared with all the controls reveals only a small increase in the yield of MN total in the radiation workers, with an increase in MNCM- scores being more pronounced, however, not significant $(\mathrm{P}=0.15)$.

Personal dosimetry analysis. The data obtained from the personal dosimetry records, the effective dose E for IRW and the personal dose equivalent $\mathrm{Hp}(10)$ for NMT, are presented in Table III. In this table these dose parameters are given as cumulative doses for periods of 12 and 36 months preceding the blood sampling. In addition, the $\mathrm{E}$ and $\mathrm{Hp}(10)$ values over 36 months corrected for the disappearance of $\mathrm{MN}$ are additionally tabulated. To this end $\mathrm{MN}$ were assumed to disappear with an in vivo half-life of 1 year (12).

Table III indicates that according to the personal dosimetry data, the NMT group received a higher radiation burden compared with the IRW group.

Comparison between biological and physical dosimetry data. Fig. 4 presents an overview of the individual data points of both groups of radiation workers. Fig. 4 presents the $\mathrm{MN}$ yields (MN total and MNCM-) as a function of the cumulative dose over 36 months, corrected for the disappearance of $\mathrm{MN}$ with a half-life of 1 year (corrected, 36 months). Fig. 4 indicates that the IRWs exhibited higher chromosomal damage according to biological dosimetry analysis using the MN-centromere assay, however, received a lower radiation burden according to the personal dosimetry records.

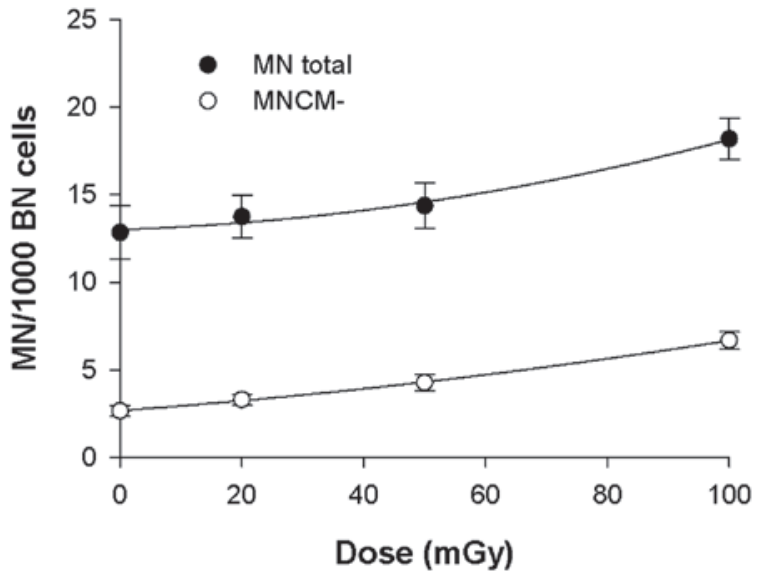

Figure 2. Dose-response curves for the MN total yield and the MNCM- yield. Dose-response curves are fitted using a weighted linear-quadratic regression. Values are presented as the mean \pm standard error on the mean of the 16 control donors. MN, micronuclei; MNCM-, centromere-negative MN; BN. binucleate.

\section{Discussion}

The first part of the present study aimed to improve a semi-automated MN centromere assay for the biological dosimetry of medical radiation workers exposed to low doses. Using a Wilcoxon signed-rank test, it was demonstrated that for small group sizes of about 10-15 individuals, the semi-automated MNCM- scoring enables the discrimination between 0 Gy and $50 \mathrm{mGy}$, while with semi-automated scoring of MN total a significant difference was only observed between 0 Gy and $100 \mathrm{mGy}$. These results indicate that MNCM- scoring markedly improved the detection capability of the assay. In earlier in vitro studies using the MN-centromere assay the lowest doses applied were 50 or $100 \mathrm{mGy}$, and the detection limit reported ranged between 50 and $100 \mathrm{mGy}(9,15,16)$.

In the present study, spontaneous $\mathrm{MN}$, present in non-irradiated samples, consisted mainly of MNCM+ (79\%), 


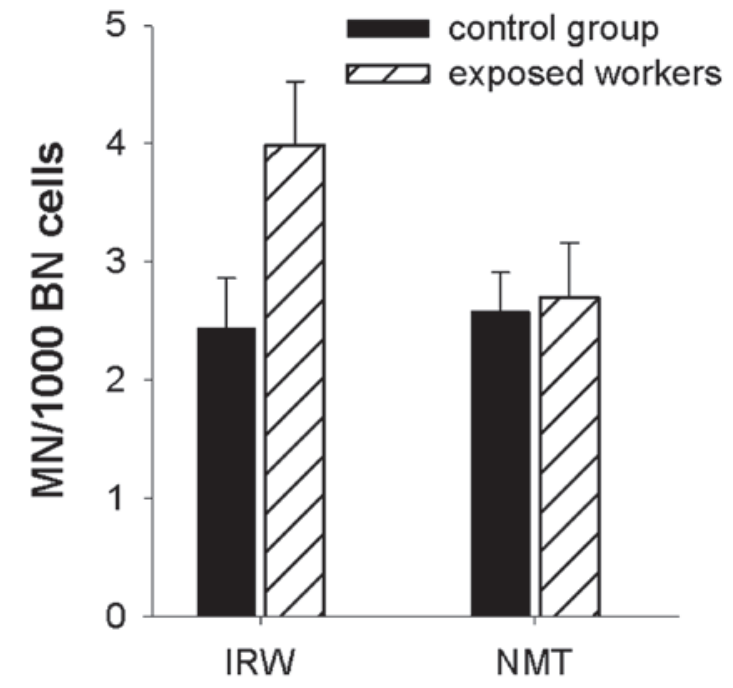

Figure 3. Comparison of the mean number of centromere-negative MN obtained from exposed radiation workers, IRW and NMT, and their matched controls. Values are presented as the mean \pm standard error of the mean. IRW, interventional radiation workers; NMT, nuclear medicine technologists; MN, micronuclei; BN, binucleate.

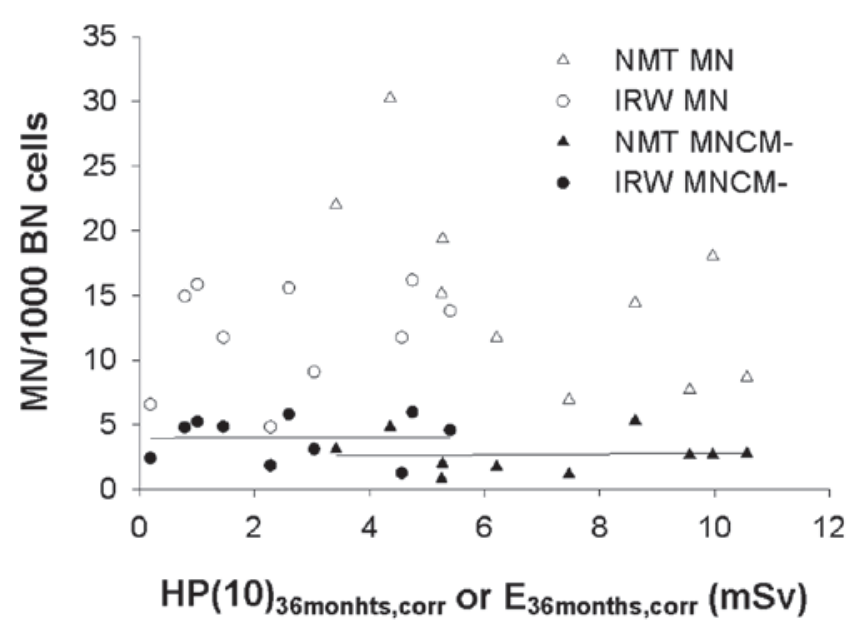

Figure 4. The individual MN yields (MN total and MNCM-) as a function of the cumulative effective dose $\mathrm{E}$ and personal equivalent dose $\mathrm{Hp}(10)$ over 36 months, corrected for disappearance of MN for the IRW and NMT groups, respectively. The lines indicate the linear regression analysis through the MNCM- data points for the IRW and NMT groups. MN, micronuclei; centromere-negative MN; IRW, interventional radiation workers; NMT, nuclear medicine technologists; BN, binucleate; corr, corrected.

with the increase in MN following low-dose exposure attributed to an increase in the number of MNCM-. These data are in line with a previous study (9). Although the MN-centromere assay is more labour-intensive compared with the standard MN assay, the use of a home-tailored pan-centromeric probe and a semi-automated scoring procedure, described here and in Baeyens et al (9), keeps the expense and scoring time reasonably low. This makes the assay suitable for large-scale applications, such as for biomonitoring. An automated procedure has additionally been developed for dicentric analysis, which is the gold standard for biological dosimetry. Though this procedure reduces the workload of the dicentric assay, it additionally reduces its sensitivity. Further investigation and validation is required before automated dicentric scoring can be applied for biomonitoring purposes (17).

In the second part of the current study, the semi-automated $\mathrm{MN}$ centromere assay was used to perform a direct comparison between exposed radiation workers (IRW and NMT) and two groups of matched controls $\left(\mathrm{CON}_{\mathrm{IRW}}\right.$ and $\left.\mathrm{CON}_{\mathrm{NMT}}\right)$.

A first analysis of the total $\mathrm{MN}$ yields indicated that there was no significant difference between the pooled exposed workers and the pooled controls. Notably, a significant difference was observed in $\mathrm{MN}$ and $\mathrm{MNCM}+$ between the two exposed and control groups applying the Kruskal-Wallis test, which may be attributed predominantly to the lower number of $\mathrm{MN}$ and $\mathrm{MNCM}+$ in the $\mathrm{CON}_{\mathrm{IRW}}$ group. It should be noted that the IRW and $\mathrm{CON}_{\text {IRW }}$ groups consist only of males while the NMT and $\mathrm{CON}_{\text {NMT }}$ groups consist of 6 females and 4 males. When the controls are divided into two groups according to gender, higher numbers of MN total and MNCM+ per 1000 $\mathrm{BN}$ cells are observed in females (females, $\mathrm{MN}$ total $=16.66$, $\mathrm{MNCM}+=14.62$; males, $\mathrm{MN}$ total $=10.28, \mathrm{MNCM}+=7.38$ ). Conversely MNCM- are comparable between these groups (2.04 vs. 2.90 MNCM-). These data confirm other findings indicating that the centromere-positive subset of $\mathrm{MN}$ is more susceptible to gender effects and is higher in females compared with males. Age may additionally be a confounding factor, and previous studies have reported that the increase of MN with age is due to an increase in centromere-positive MN. In the present study, the confounding effect of age on the MN yields may be excluded, as the mean age in both the exposed and control groups were comparable, as all worker/control pairs were age-matched. The effects of age and gender on MNCM+ have been extensively described in Baeyens et al (9). In addition, it has been reported that the $\mathrm{X}$-chromosome is often involved in spontaneous chromosome loss (18).

As the majority of spontaneous $\mathrm{MN}$ are $\mathrm{MNCM}+$, scoring of only the MNCM- subset removes the 'background noise' represented by $\mathrm{MNCM}+$, and considerably lowers the detection threshold of the MN assay for the in vivo biomonitoring of radiation workers exposed to low radiation doses, according to their personal dosimetry records. Using telomeric and centromeric FISH staining, Lindberg et al (19) demonstrated that the majority of the centromere-negative MN harbour terminal acentric fragments, supporting the observation that centromere-negative MN are a better indicator of radiation-induced damage.

In a number of studies where the standard MN assay was used to biomonitor radiation workers, heterogeneous and contradictory results have been reported (2). Ropolo et al (2) state that the MN content is not the sole factor responsible for the heterogeneity in the results. One important factor is that in the majority of MN studies, mixed populations of hospital staff are investigated. Only in few studies (including the present), is the MN analysis performed in specific groups of radiation workers, such as those working in nuclear medicine and interventional cardiology, and who receive the highest levels of exposures. Another important factor responsible for the heterogeneous results of many studies is the way the personal dosimetry data are used as an exposure parameter. In certain studies, the estimated dose is based on personal dosimetry readings during the previous 12 months, the previous 5-10 years or based on the accumulated dose during the entire time of employment. Only in few studies is a correction performed for 
the disappearance of MN. In the current study, the personal doses registered during the previous 12 or 36 months, with or without a correction for $\mathrm{MN}$ disappearance were used as occupational exposure quantities.

Analysis of the centromere-negative $\mathrm{MN}$ in all exposed workers compared with control individuals indicated an increased number of MNCM- in the exposed group, however, this increase was not significant $(\mathrm{P}=0.15)$. When the exposed group was divided into two groups, based on the specific professional activities, a significantly increased MNCMnumber was observed in the IRW group compared with their controls $(\mathrm{P}=0.05)$. A significant increase of MNCM- scores was not observed in the NMT group, indicating that the IRWs are exposed to higher levels of occupational radiation compared with the NMT group. The higher yield of MNCMin the IRW group compared with the NMT group is however, not in agreement with the personal dosimetry records of both groups. Dose estimates for the IRW group are considerably lower than in the NMT group. In the IRW group, the effective dose E, deduced from double dosimetry readings, was used as the personal dose. For this calculation an algorithm was applied, which takes into account the organs and organ weighting factors of the ICRP 103 publication: $\mathrm{E}=0.79 \mathrm{Hp}, \mathrm{u}(10)$ $+0.100 \mathrm{Hp}, \mathrm{o}(10)$ (10). A number of studies have already been published dealing with the $\alpha$ and $\beta$ coefficients in the $E=\alpha$ $\mathrm{Hp}, \mathrm{u}(10)+\beta \mathrm{Hp}, \mathrm{o}(10)$ expression. An overview of these coefficients indicates that the majority of algorithms adopt $\alpha$ values close to 1 and $\beta$ values in the range $0.07-0.10(20,21)$. Utilizing a different algorithm in the present study will not alter the $\mathrm{E}$ values for the IRW group to a large extent.

The discrepancies observed between the physical dose estimates and the biological effect in both groups of radiation workers may indicate the limitations of physical dosimetry, in particular in interventional radiology/cardiology. This has been reported in previous studies $(1,2,7)$. Although in interventional radiology/cardiology, double dosimetry practice is recommended and legally required in Belgium and other countries, its application in daily practice is not granted. For example, interventional radiologists/cardiologists do not always wear their (or both) dosimeters during interventions performed at different locations. Personal dosimeters are not always worn correctly at the right position; hence they are not representative of the exposure. In certain cases, dosimeters remain attached to the apron, meaning the dose is no longer personal. In the current study of interventional radiologists/cardiologists, no readings were obtained for the dosimeters worn under the apron in 102 of 300 reading months, and for dosimeters worn above the apron in 46/270 reading months. Another consideration is that no consensus exists on the most suitable algorithm to calculate the effective dose from the two readings. The lower frequency of MNCM- observed in NMTs compared with IRWs may be associated with the personal dosimetry records in NMTs not wearing lead aprons and related to annihilation photons, which are more representative of the real radiation burden in these workers. Finally, it should be considered that the cytogenetic effects are associated with the blood dose and not the personal equivalent dose, $\mathrm{Hp}(10)$, and the effective dose, E, quantities which serve as tools to monitor radiation workers.

The present study demonstrates that a semi-automated $\mathrm{MN}$-centromere assay is suitable for large-scale low dose biomonitoring. The observations of enhanced frequencies of centromere-negative MN, representing chromosomal damage, in medical radiation workers exposed to doses within the ICRP regulatory limits, according to their personal dosimetry records, further supports that chronic radiation exposure may be hazardous due to enhanced genotoxicity. These results indicate that any suggestions that the current dose limits are unnecessarily low and restrictive and could be raised, should be rejected.

The present study strengthens the importance of cytogenetic analysis performed together with physical dosimetry, as a routine biomonitoring method for medical workers receiving the highest occupational radiation burden. The disagreement between the differences in personal dosimetry data and cytogenetic results of the two groups of medical radiation workers may be associated with the fact that the application of double dosimetry in the daily practice of interventional X-ray workers is not evident, reducing the reliability of the resulting effective doses.

\section{Acknowledgements}

The authors wish to thank Ms. Toke Thiron, Ms. Virginie De Gelder, Ms. Johanna Aernoudt and Ms. Greet De Smet of the Department of Basic Medical Sciences of Ghent University for their excellent practical assistance, and additionally, the workers and volunteers who participated in the current study. The present study was funded by the CESI Scientific Fund Occupational Health Service.

\section{References}

1. Zakeri F and Hirobe T: A cytogenetic approach to the effects of low levels of ionizing radiations on occupationally exposed individuals. Eur J Radiol 73: 191-195, 2010.

2. Ropolo M, Balia C, Roggieri P, Lodi V, Nucci MC, Violante FS, Silingardi $\mathrm{P}$, Colacci A and Bolognesi C: The micronucleus assay as a biological dosimeter in hospital workers exposed to low doses of ionizing radiation. Mutat Res 747: 7-13, 2012.

3. Martin CJ, Sutton DG, West CM and Wright EG: The radiobiology/radiation protection interface in healthcare. J Radiol Prot 29: A1-A20, 2009.

4. Thierens H, Vral A, Morthier R, Aousalah B and De Ridder L: Cytogenetic monitoring of hospital workers occupationally exposed to ionizing radiation using the micronucleus centromere assay. Mutagenesis 15: 245-249, 2000.

5. Cardoso RS, Takahashi-Hyodo S, Peitl P Jr, Ghilardi-Neto T and Sakamoto-Hojo ET: Evaluation of chromosomal aberrations, micronuclei and sister chromatid exchanges in hospital workers chronically exposed to ionizing radiation. Teratog Carcinog Mutagen 21: 431-439, 2001

6. Dias FL, Antunes LM, Rezende PA, Carvalho FE, Silva CM, Matheus JM, Oliveira JV Jr, Lopes GP, Pereira GA and Balarin MA: Cytogenetic analysis in lymphocytes from workers occupationally exposed to low levels of ionizing radiation. Environ Toxicol Pharmacol 23: 228-233, 2007.

7. Montoro A, Rodríguez P, Almonacid M, Villaescusa JI, Verdú G, Caballín MR, Barrios L and Barquinero JF: Biological dosimetry in a group of radiologists by the analysis of dicentrics and translocations. Radiat Res 164: 612-617, 2005.

8. International Atomic Energy Agency (IAEA) Emergency Preparedness and Response Biodosimetry 2011: Cytogenetic Dosimetry: Applications in preparedness for a response to radiation emergencies. IAEA, Vienna, Austria (2011).

9. Baeyens A, Swanson R, Herd O, Ainsbury E, Mabhengu T, Willem P, Thierens H, Slabbert JP and Vral A: A semi-automated micronucleus-centromere assay to assess low-dose radiation exposure in human lymphocytes. Int J Radiat Biol 87: 923-931, 2011. 
10. Von Boetticher H, Lachmund J and Hoffmann W: An analytic approach to double dosimetry algorithms in occupational dosimetry using energy dependent organ dose conversion coefficients. Health Phys 99: 800-805, 2010.

11. International Commission on Radiological Protection (ICRP): The 2007 Recommendations of the International Commission on Radiological Protection. ICRP publication 103, Ann ICRP 37: 2-4 (2007)

12. Thierens H, De Ruyck K, Vral A, de Gelder V, Whitehouse CA, Tawn EJ and Boesman I: Cytogenetic biodosimetry of an accidental exposure of a radiological worker using multiple assays. Radiat Prot Dosimetry 113: 408-414, 2005.

13. Willems P, August L, Slabbert J, Romm H, Oestreicher U, Thierens $\mathrm{H}$ and Vral A: Automated micronucleus (MN) scoring for population triage in case of large scale radiation events. Int $\mathbf{J}$ Radiat Biol 86: 2-11, 2010.

14. Schunck C, Johannes T, Varga D, Lörch T and Plesch A: New developments in automated cytogenetic imaging: Unattended scoring of dicentric chromosomes, micronuclei, single cell gel electrophoresis, and fluorescence signals. Cytogenet Genome Res 104: 383-389, 2004.

15. Wojcik A, Kowalska M, Bouzyk E, Buraczewska I, Kobialko G, Jarocewicz $\mathrm{N}$ and Szumiel I: Validation of the micronucleus-centromere assay for biological dosimetry. Gen Mol Biol 23: 1083-1085, 2000.
16. Pala FS, Alkaya F, Tabakcioglu K, Tokatli F, Uzal C, Parlar S and Algünes C: The effects of micronuclei with whole chromosome on biological dose estimation. Turkish J Biol 32: 283-290, 2008.

17. Romm H, Ainsbury E, Barnard S, Barrios L, Barquinero JF, Beinke C, Deperas M, Gregoire E, Koivistoinen A, Lindholm C, et al: Automatic scoring of dicentric chromosomes as a tool in large scale radiation accidents. Mutat Res 756: 174-183, 2013.

18. Norppa H and Falck GC: What do human micronuclei contain? Mutagenesis 18: 221-233, 2003.

19. Lindberg HK, Falck GC, Järventaus H and Norppa H: Characterization of chromosomes and chromosomal fragments in human lymphocyte micronuclei by telomeric and centromeric FISH. Mutagenesis 23: 371-376, 2008

20. Järvinen $\mathrm{H}$, Buls $\mathrm{N}$, Clerinx $\mathrm{P}$, Jansen $\mathrm{J}$, Miljanić $\mathrm{S}$, Nikodemová D, Ranogajec-Komor M and d'Errico F: Overview of double dosimetry procedures for the determination of the effective dose to the interventional radiology staff. Radiat Prot Dosimetry 129: 333-339, 2008.

21. Järvinen $H$, Buls N, Clerinx P, Miljanic S, Nikodemová D, Ranogajec-Komor M, Struelens L and d'Errico F: Comparison of double dosimetry algorithms for estimating the effective dose in occupational dosimetry of interventional radiology staff. Radiat Prot Dosimetry 131: 80-86, 2008. 